\title{
Mittäterschaft beim Fahrlässigkeitsdelikt im Strafrecht
}

Gleichzeitig eine Besprechung des Urteils des Bundesgerichts 6B_360/2016, 6B_361/2016 (zur Publikation vorgesehen) vom 1. Juni 2017

Daniel Häring *

Die fahrlässige Mittäterschaft ist im Strafrecht spätestens seit dem Rolling-StonesFall fester Bestandteil der Diskussionen rund um das Fahrlässigkeitsdelikt. Während die traditionelle Lehre sich dieser Rechtsfigur eher skeptisch gegenüberstellt, lässt sich in jüngerer Zeit ein Trend in Richtung Anerkennung einer Mittäterschaft beim Fahrlässigkeitsdelikt ausmachen. Der durch das Bundesgericht gefällte Raketenfall ist Anlass der vorliegenden Untersuchung, welche die Diskussion aufnimmt, die Argumente gegen die Rechtsfigur kritisch beleuchtet und ein Konzept für eine Mittäterschaft beim Fahrlässigkeitsdelikt vorstellt.

I. Das wiederkehrende Problem

1. Zwei Bundesgerichtsfälle mit unterschiedlichen Urteilen....................................2

2. Ähnlich gelagerte Sachverhalte..........................................................................

II. Bisherige Lösungsansätze und deren Schwachstellen ........................................................

1. Vorab: Wäre es ein Vorsatzdelikt, gäbe es keine Probleme ........................................

2. Freispruch in dubio pro reo ..................................................................................

3. Einheitstäterschaft - Mitverursachungstheorie ..........................................................

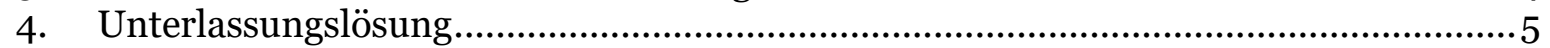

5. Die Lösung des Bundesgerichts im Rolling-Stones-Fall.............................................6

III. Argumente gegen eine fahrlässige Mittäterschaft und deren Relativierung......................7

1. Kein gemeinsamer Tatentschluss? ...........................................................................

2. Keine gesetzliche Grundlage für eine fahrlässige Mittäterschaft?.............................8

3. Keine Möglichkeit der Unterscheidung von Beteiligungsrollen? ................................8

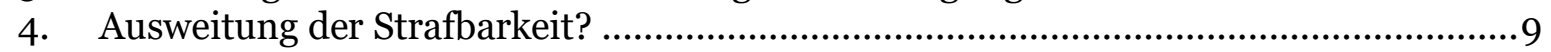

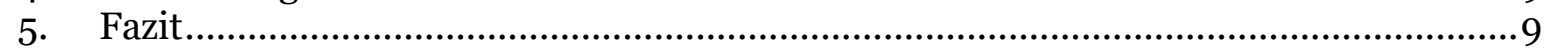

IV. (Tatbestands-)Elemente einer Mittäterschaft beim Fahrlässigkeitsdelikt ........................9

V. Das Urteil des Bundesgerichts im Lichte der Mittäterschaft

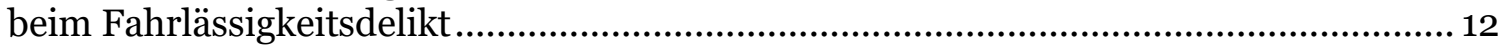

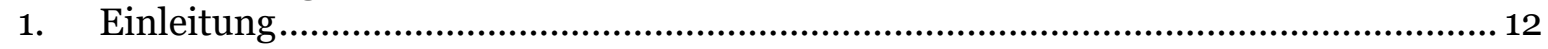

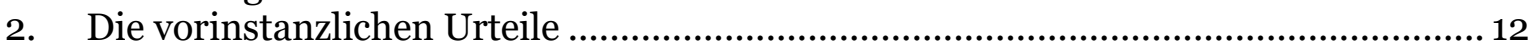

3. Das Urteil des Bundesgerichts in der Sache ............................................................... 13

4. Analyse des Urteils mit Blick auf die fahrlässige Mittäterschaft ................................... 14

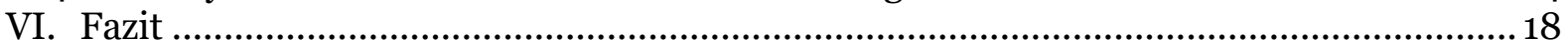

Zitiervorschlag: Daniel Häring, Mittäterschaft beim Fahrlässigkeitsdelikt im Strafrecht, in: sui-generis 2018, S. 1

URL: $\quad$ sui-generis.ch/55

DOI: $\quad$ https://doi.org/10.21257/sg.55

* Dr. iur. Daniel Häring, Rechtsanwalt, Partner bei böckli bühler partner, Basel; Lehrbeauftragter für Strafrecht an der Universität Basel; Vizepräsident des Zivilkreisgerichts Basellandschaft Ost.

Dieses Werk ist lizenziert unter einer Creative Commons Namensnennung - Weitergabe unter gleichen Bedingungen 4.0 International Lizenz. 


\section{Das wiederkehrende Problem}

\section{Zwei Bundesgerichtsfälle mit un- terschiedlichen Urteilen}

1 Das schweizerische Bundesgericht hatte am 1. Juni 2017 den folgenden Sachverhalt zu beurteilen, der in der vorliegenden Untersuchung als Ausgangsfall dienen soll: ${ }^{1}$

Am 1. Januar 2013 ging um oo.o5 Uhr bei der Einsatzzentrale der Kantonspolizei Aargau die Meldung ein, dass ein Balkon eines Mehrfamilienhauses in Flammen stand. A und $B$ wurden über ein Ausschlussverfahren als Täter ermittelt. Die beiden haben kurz vor Mitternacht Raketen abgefeuert. Eine der abgefeuerten Raketen war in ihrer Flugbahn fehlgeleitet und verursachte den Brand. Es war nicht mehr zu ermitteln, welcher der beiden Beteiligten die fehlgeleitete Rakete zündete, wenngleich es einer von beiden gewesen sein musste. Die Fehlleitung der Rakete hatte ihre Ursache in einem unsachgemässen Abfeuern («falsches» In-den-Boden-Stecken). Es stand fest, dass die beiden Beschuldigten zwar gemeinsam beschlossen hatten, die Raketen zu zünden, es wurde aber keine gemeinsame (explizite) Absprache in Bezug auf das «falsche» bzw. «gefährliche» Abfeuern festgestellt. Es entstand Sachschaden in der Höhe von CHF 868'951.-, Personen wurden keine verletzt.
2 Schon vor rund dreissig Jahren ereignete sich Ähnliches. Das Bundesgericht hatte am 15. Mai 1987 einen Fall zu entscheiden, der in die schweizerische Strafrechtsgeschichte als «Rolling-StonesFall» einging: ${ }^{2}$

$A$ und $B$ bemerkten neben der Strasse am rechten Tössufer zwei grosse Steinbrocken, welche sie auf Anregung von A den dortigen Abhang hinunterzurollen beabsichtigten. Ihnen waren einerseits die örtlichen Verhältnisse bestens bekannt und sie wussten insbesondere, dass sich in jenem Bereich am Tössufer öfters Leute - vorwiegend Fischer - aufhielten. Andererseits war ihnen auch bewusst, dass mit den grossen Steinen von ca. $52 \mathrm{~kg}$ bzw. über $100 \mathrm{~kg}$ Gewicht eine Person, die sich zufällig im Gefahrenbereich aufhält, getroffen werden könnte. Aus diesen Gründen ging B auf Vorschlag von A ein paar Schritte nach vorne gegen den Abgrund, um abzuklären, $o b$ sich jemand unten am Abhang bzw. im Bereich des Tössufers aufhalte. Dabei rief er einmal laut hinunter, ob jemand unten sei, wobei er aber von seinem Standort aus das rechte Tössufer nicht einsehen konnte. Nachdem aufdas Rufen niemand geantwortet hatte, kehrte $B$ zи A zurück, behändigte den grossen, über $100 \mathrm{~kg}$ schweren Stein und liess ihn den Abhang hinunterrollen. Unmittelbar nachher rollte A den kleineren, ca. $52 \mathrm{~kg}$ schweren Stein ebenfalls hinunter. Es steht fest, dass der unter dem Abhang befindliche Fischer $X$ von einem der beiden Steine getroffen wurde; jedoch konnte nicht geklärt werden, von welchem.
Urteil des Bundesgerichts 6B_360/2016, 6B_361/2016 vom 1. Juni 2017.
BGE 113 IV 58. 

welche das Bundesgericht in den beiden Fällen erlassen hat. Während im RollingStones-Fall beide Beteiligten wegen fahrlässiger Tötung verurteilt wurden, wurden im Raketenfall beide vom Vorwurf der fahrlässigen Verursachung einer Feuersbrunst freigesprochen. 3

\section{2. Ähnlich gelagerte Sachverhalte}

4 Die Liste ähnlicher Fälle lässt sich verlängern, etwa wenn zwei Einbrecher eine Fabrikhalle mittels Streichhölzer ausleuchten, diese achtlos wegwerfen, und die Halle zu brennen beginnt, ohne dass sich im Nachhinein eruieren lässt, wer das letztlich brandstiftende Streichholz geworfen hat.4 Oder trifft ein aus drei oder mehr Personen bestehender Verwaltungsrat den Beschluss, ein Produkt um dessen Gefährlichkeit die Beteiligten wissen könnten - nicht vom Markt zu ziehen, kann sich jeder im Falle von Fahrlässigkeitserfolgen darauf berufen, seine Gegenstimme hätte nichts am Beschluss geändert. 5

5 Die Problematik bei der strafrechtlichen Aufarbeitung dieser und ähnlich gelagerter Fälle ist stets dieselbe, sie liegt in der natürlichen Kausalität. Es ist zwar jeweils erstellt, dass die Handlungen sämtlicher Beteiligter gemeinsam zu den jeweiligen Fahrlässigkeitserfolgen geführt haben. Wird allerdings die natürliche Kausalität der Einzelhandlung jeder Person mithilfe der einschlägigen Conditio-sine-qua-

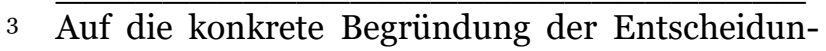
gen wird weiter hinten (vgl. V. 3.) detailliert eingegangen.

4 OLG Schleswig, NStZ 1982, S. 116 f.; sehr ähnlich BayObLG, NJW 1990, S. 3032.

5 So (verkürzt) die Ausgangslage im deutschen «Lederspray-Fall», BGHSt 37, $106 \mathrm{ff}$.
non-Formel ${ }^{6}$ geprüft, ist diese zu verneinen. So ist etwa im Ausgangsfall zwar erstellt, dass das gemeinsame Raketenabfeuern den Brand verursacht hat. Wird jedoch geprüft, ob A oder B je einzeln für den Brand mit an Sicherheit grenzender Wahrscheinlichkeit eine kausale Ursache gesetzt haben, ist dies zu verneinen. Die Bestimmung der Kausalität bereitet also dort Schwierigkeiten, wo mehrere Ursachen hätten erfolgsursächlich sein können, ex post aber nicht festzustellen ist, welche.

\section{Bisherige Lösungsansätze und deren Schwachstellen}

\section{Vorab: Wäre es ein Vorsatzdelikt, gäbe es keine Probleme}

6 Ginge es in den genannten Fällen um Vorsatzdelikte, bestünden strafrechtlich keine Zurechnungsprobleme. Beim Vorsatzdelikt wird diese Problematik der fehlenden Einzelkausalität bei gemeinsamer Tatbegehung seit jeher mittels der Figur der Mittäterschaft befriedigend gelöst. Jedem Mittäter werden auch die Tatbeiträge der anderen zugerechnet. Somit wird nicht mehr danach gefragt, ob ein Mittäter den Taterfolg alleine verursacht hat, sondern ob ihn die Mittäter gemeinsam verursacht haben. ${ }^{7}$

$6 \overline{\text { (Natürlich) Kausal für den Deliktserfolg ist nach }}$ dieser Formel eine Handlung, wenn sie nicht hinweggedacht werden kann, ohne dass der konkrete Erfolg entfiele; vgl. etwa BGE 125 IV 195, 197; BGE 122 IV 17, 23.

7 So ausdrücklich der vorliegend zu besprechende Entscheid Urteil des Bundesgerichts 6B_36o/2016, 6B_361/2016 vom 1. Juni 2017, E. 4.10. Vgl. auch Basler Kommentar Strafrecht I, Art. 1-110 StGB und Jugendstrafgesetz, Niggli/Wiprächtiger (Hrsg.), 3. Aufl., Basel 2013 (nachfolgend zit.: BSK StGB-Bearbeiter), Marc Forster, Vor. Art. $24 \mathrm{~N} 8$ sowie N 24; BGE 118 IV 227, 232. Schiessen etwa zwei Personen aufgrund 
7 Bei fahrlässiger Tat hingegen fehlt es zumindest nach traditioneller Lehre - bis heute an einer entsprechenden Möglichkeit, da es nach dieser Lehre beim Fahrlässigkeitsdelikt keine Mittäterschaft geben soll. ${ }^{8}$ Deshalb werden die streitgegenständlichen Fälle auf unterschiedlichste andere Art gelöst. $\mathrm{Zu}$ den wichtigsten Lösungsansätzen gehören die nachfolgenden. 9

\section{Freispruch in dubio pro reo}

8 Der erste Lösungsansatz ist einfach: Freispruch sämtlicher Beteiligter in dubio

eines gemeinsamen Tatplans gemeinsam auf ihr Opfer, trifft aber nur eine Kugel, werden regelmässig beide Personen als Mittäter erfasst und wegen der Tötung verurteilt.

8 Gegen die fahrlässige Mittäterschaft, mit unterschiedlichen Begründungen, etwa Bernhard Corboz, Commentaire Romand, Code pénal I, Basel 2009, Art. 12 N 180; Andreas Donatsch, Mittäterschaft oder Teilnahme am fahrlässigen Erfolgsdelikt, SJZ 1989, S. 109 ff., S. 111; derselbe, StGB Kommentar, Donatsch et al. (Hrsg.), 19. A. Zürich 2013, Art. $12 \mathrm{~N} \mathrm{28;} \mathrm{Andreas} \mathrm{Do-}$ natsch/Brigitte Tag, Strafrecht I, Verbrechenslehre, 9. Aufl., Zürich 2013, S. 370; Philippe Spitz, Strafrechtliche Produkthaftung - Übertragbarkeit zivilrechtlicher Betrachtungsweisen?, Diss. Basel 2000, Basel/Genf/München 2001, S. 79, 452 f.; Günter Stratenwerth, Schweizerisches Strafrecht, Allgemeiner Teil I: Die Straftat, 4. A. Bern 2011, § 16 N 49; Stefan Trechsel/Peter Noll/Mark Pieth, Schweizerisches Strafrecht Allgmeiner Teil I, 7. A. Zürich 2017, S. 198 ff.; Hans Walder, The rolling stones - Urteilsanmerkung Strafrecht, recht 1989, S. 56 ff. Auch Andreas Eicker/Sonja Mango-Meier, Repetitorium Nebenstrafrecht SVG und BetmG, Zürich 2016, S. 43, und Trechsel/Jean-Richard, Schweizerisches Strafgesetzbuch, Praxiskommentar, Trechsel/ Pieth (Hrsg.), 2. A. Zürich/St. Gallen 2012, Art. $12 \mathrm{~N} \mathrm{44}$, sind der Auffassung, bei einer fahrlässigen Tatbegehung falle Mittäterschaft ausser Betracht, erläutern jedoch nicht näher, warum dies so sein soll.

9 Vgl. für eine umfassende Darstellung der möglichen Lösungsansätze mit kritischer Würdigung vgl. auch Daniel Häring, Die Mittäterschaft beim Fahrlässigkeitsdelikt, Diss. Basel, Basel/Genf/ München 2005, S. 21 ff.; sowie derselbe, Das verhängnisvolle Streichholz, ius.full 2/2008, S. $64 \mathrm{ff}$., S. $67 \mathrm{ff}$. pro reo. Nach dieser Auffassung sei in Fällen zweifelhafter Kausalität zu Gunsten jedes Beteiligten anzunehmen, der jeweils andere habe die Erfolg verursachende Handlung ausgeführt. Wenn somit keinem der Beteiligten der Erfolg mit der notwendigen Wahrscheinlichkeit zugerechnet werden kann, müssen alle freigesprochen werden. ${ }^{10}$

9 Diese Lösung ist rechtlich vertretbar, lässt jedoch das den jeweiligen Fällen inhärente Strafbedürfnis ausser Acht. Die Handlungen der Beteiligten, also etwa das unsorgfältige Raketenabfeuern oder das unsorgfältige Steinewerfen, sind weder sozial nützlich noch sozial üblich, weshalb eine fahrlässige Nebenfolge derselben nur schwer als sozialadäquat eingestuft werden kann. Eine Bestrafung beider erscheint deshalb gerecht, selbst wenn nachgewiesen werden könnte, wer die verhängnisvolle Rakete abgefeuert oder wer den verhängnisvollen Stein geworfen hat. Hätten die beiden Beteiligten bei gleicher Sachlage mit einem gemeinsamen Tatentschluss die Raketen gestartet, um den Brand vorsätzlich zu verursachen, würde jedenfalls ein Freispruch beider in dubio pro reo wohl kaum in Betracht gezogen werden. ${ }^{11}$

\section{Einheitstäterschaft - Mitverursachungstheorie}

10 Ein weiterer Lösungsansatz umgeht das Kausalitätsproblem, indem jeder irgendwie geartete erfolgs(mit)ursächliche Bei-

10 Dafür etwa Trechsel/Noll/Pieth (Fn. 8), S. 200; Philippe Spitz (Fn. 8), S. 453 und wohl Donatsch/ Tag (Fn. 8), S. 371.

11 Daniel Häring (Fn. 9, 2005), S. 22 f.; derselbe (Fn. 9, 2008), S. 68; kritisch auch Christof Riedo/Michaela Chvojka, Fahrlässigkeit, Mittäterschaft und Unsorgfaltsgemeinschaft, ZStrR 2002, S. 152 ff., S. 163, Fn. 39 . 
trag für die Bejahung der Fahrlässigkeitshaftung als ausreichend erklärt wird. Die Theorie der Einheitstäterschaft beim Fahrlässigkeitsdelikt besagt, dass jedes irgendwie geartete sorgfaltswidrige und erfolgsmitursächliche Handeln zur Fahrlässigkeitstäterschaft führt. Nach dieser Theorie ist in Fällen unklarer Kausalität bereits als Erfolgsursache ausreichend, dass der eine Täter den anderen durch sein Mittun in dessen Handeln bestärkt und unterstützt hat. Auch wenn die Personen gemeinsam Handeln, werden sie - zum Teil unter dem Begriff «Nebentäter» - nach dieser Auffassung als Einzeltäter behandelt. ${ }^{12}$

Auch diese Lösung hat mit Schwierigkeiten zu kämpfen. Es ist unklar, was A (und somit auch B) eigentlich genau vorgeworfen wird. Die Theorie der Einheitstäterschaft präsentiert eine Auswahlsendung: entweder hat A selbst die verhängnisvolle Rakete gezündet, oder wenn nicht, hat er wenigstens die Handlungen des B (psychisch) unterstützt, und zwar durch den gemeinsamen Entschluss des Raketenabfeuerns, oder durch die blosse Anwesenheit am Tatort, oder durch das Zünden der eigenen Rakete. Eine solche «Wahlfeststellung» kann zur strafrechtlichen Zurechnung nur schwerlich ausreichen. Die Lösung ist zu konturenlos, da die Grenzen einer strafwürdigen Mitverursachung nicht ausreichend feststehen. ${ }^{13}$

$12 \overline{\text { So etwa Günter Stratenwerth (Fn 8), § } 16 \text { N } 49 .}$. Für den Rolling-Stones-Fall auch BSK StGBNiggli/Maeder (Fn 7), Art. 12 N. 135 und Bernhard Sträuli, Commentaire Romand, Code pénal I, Basel 2009, Intro aux art. 24 à $27 \mathrm{~N}$ 116, die im Übrigen aber zur fahrlässigen Mittäterschaft eine differenzierende Auffassung vertreten.

13 So bereits Bettina Weisser, Gibt es eine fahrlässige Mittäterschaft?, JZ 1998, S. 230 ff., 235; kritisch auch Daniel Häring (Fn. 9, 2005), S. 25 ff.; derselbe (Fn. 9, 2008), S. 68 f.
12 Ein solcher Ansatz verstösst zudem gegen das Verantwortungsprinzip. Ausserhalb des Unterlassungsdelikts ist jeder grundsätzlich nur für eigenes Handeln verantwortlich, nicht für fremdes. Es ist deshalb fraglich, ob jemand wirklich als Täter zu bestrafen ist, wenn er mit der ihm vorgeworfenen Handlung die Haupthandlung des Täters lediglich unterstützt oder veranlasst. Im Bereich der Vorsatzdelikte ist unbestritten, dass solch untergeordnete Handlungen lediglich als Teilnahme zu werten sind. Weshalb beim Fahrlässigkeitsdelikt hingegen auch untergeordnete Handlungen zur Täterschaft führen sollen, ist bisher nicht überzeugend dargelegt worden. ${ }^{14}$

\section{Unterlassungslösung}

13 Schliesslich wird in Fällen wie den vorliegenden als Lösung auch vertreten, den Vorwurf einfach $\mathrm{zu}$ verlagern, weg vom aktiven Handeln, hin zu einem Unterlassen. Dem jeweils Beteiligten wird dabei vorgeworfen, es unterlassen zu haben, den anderen von seinem pflichtwidrigen Tun abzuhalten. ${ }^{15}$ Im Ausgangsfall würde A somit nicht mehr vorgeworfen, selbst die verhängnisvolle Rakete gezündet zu haben, sondern nichts unternommen $\mathrm{zu}$ haben, B von dessen Raketenzünden abzuhalten. A könnte dadurch als Unterlassungstäter verurteilt werden; gleiches würde für B gelten.

14 Diese Betrachtungsweise widerspricht dem tatsächlichen Geschehensablauf und wirkt unnatürlich. Der Schwerpunkt des

$14 \overline{\text { Daniel Häring (Fn. 9, 2005), S. } 26 \text { f.; derselbe }}$ (Fn. 9, 2008), S. 68 f.

15 BSK StGB-Forster (Fn. 7), Vor Art. 24 N 26; Hans Walder (Fn. 8), S. 57 ff.; und in einem sehr ähnlich gelagerten Fall in Deutschland vom Oberlandgericht Bayern so entschieden - OLG Bayern, NJW 1990, S. 3032. 
strafrechtlichen Vorwurfs wird auf ein dem Begehen vorgelagertes Unterlassen verschoben, welches für sich allein noch keine unmittelbare Gefahr begründet. Der relevante Vorwurf im Ausgangsfall liegt kaum in der Nichtverhinderung des fremden Raketenzündens, sondern dem tatsächlichen Geschehen entsprechend - im Zünden der eigenen Rakete(n). Zudem hat in Fällen, bei denen sowohl ein Handlungs- als auch ein Unterlassungsmoment vorliegt, das Unterlassen hinter dem Handeln grundsätzlich zurückzutreten (Subsidiaritätstheorie). ${ }^{16}$ Die Unterlassungslösung muss sich vorwerfen lassen, zur Umgehung des Beweisproblems ein Handlungsdelikt in ein Unterlassungsdelikt umzudeuten und ist bereits deshalb abzulehnen. ${ }^{17}$

Unklar ist weiter, wie im Ausgangsfall die für das Unterlassungsdelikt notwendige Garantenstellung des Täters begründet werden soll. Denkbar wäre die Garantenstellung aus Ingerenz. ${ }^{18}$ Dabei ist aber fraglich, welches vorangegangene gefährliche Tun die Gefahrverantwortung begründen soll: Ist es der Entschluss zum Abfeuern von Raketen, oder aber erst das Abfeuern selbst? Es ist jedenfalls nicht schlüssig nachvollziehbar, warum das Abfeuern der eigenen Rakete für A eine Verhinderungspflicht betreffend die Handlungen des B begründen sollen. ${ }^{19}$

$16 \overline{\text { Vgl. statt vieler BGE } 129 \text { IV 119, 122; BGE } 120 \text { IV }}$ 265, 271.

17 So auch Philippe Spitz (Fn. 8), S. 82; Bettina Weisser (Fn. 13), S. 236; vgl. bereits Daniel Häring (Fn. 9, 2008), S. 69.

18 Vgl. etwa BSK StGB-Forster (Fn. 7), Vor Art. 24 $\mathrm{N} 26$.

19 Eine solche Auffassung würde eine Garantenstellung für eigenverantwortliches gefährliches Handeln anderer Personen konstruieren. Dem kann, namentlich bei Sicherungspflichten wie in casu, nur bedingt gefolgt werden; kritisch auch Bettina Weisser (Fn. 13), S. 236.
Auf jeden Fall liegt darin keine dem Unterlassen vorgelagerte Handlung, da durch das Abfeuern der Raketen der massgebliche Erfolg bereits unmittelbar und vollständig eingetreten ist. ${ }^{20}$

\section{Die Lösung des Bundesgerichts im Rolling-Stones-Fall}

16 Im Rolling-Stones-Fall verurteilte das Bundesgericht beide Steineroller wegen fahrlässiger Tötung des Fischers. Das Bundesgericht entschied derart wohltuend prägnant, ${ }^{21}$ dass die massgebliche Stelle hier im Volltext wiedergegeben wird:

«Vorliegendenfalls steht fest, dass beide Angeklagten gemeinsam die beiden Steine den Abhang hinunterrollen lassen wollten. Bei einer derartigen Konstellation ist nicht danach zu fragen, ob der jeweilige Einzelbeitrag für den tatbestandsmässigen Erfolg kausal geworden ist, sondern ob die Kausalität zwischen der gemeinsam vorgenommenen Gesamthandlung und dem eingetretenen Erfolg zu bejahen ist. Jedenfalls muss dies gelten, wenn, wie vorliegendenfalls, die sorgfaltswidrige Handlung gemeinsam beschlossen und in der Folge in einem nahen

20 So auch das OLG Schleswig, NStZ 1982, S. 116 f., in einem ähnlich gelagerten Fall. Vgl. dazu auch Daniel Häring (Fn. 9, 2005), S. 35 ff.; derselbe (Fn. 9, 2008), S. $69 \mathrm{f}$.

21 Der ehemalige Bundesrichter Martin Schubart schreibt heute dazu: «Das Bundesgericht kam mit einer kurzen Begründung, auf die ich im Lichte der Geschwätzigkeit heutiger Urteile heute noch stolz bin, etwas vereinfacht zum Ergebnis, dass hier eine Art fahrlässige Mittäterschaft vorliege, weshalb die ungeklärte Beweisfrage keine Rolle spiele und beide Angeklagten der fahrlässigen Tötung schuldig seien.» Martin Schubart, Gedanken über Freiheit und Unabhängigkeit, Anwaltsrevue 2013, S. 366 ff., S. 368. 
örtlichen und zeitlichen Zusammenhang gemeinsam durchgefuiht wird, wobei es der zufälligen Arbeitsteilung überlassen bleibt, wer welchen Stein ins Rollen bringt. Ist aber davon auszugehen, dass jedenfalls einer der beiden Steine den Tod des Opfers bewirkt hat, genügt dies zur Feststellung, dass das Verhalten des Beschwerdeführers für den eingetretenen Tod kausal geworden ist. Anders zu entscheiden wäre dann, wenn die beiden Angeklagten unabhängig voneinander gehandelt hätten.»22

Entscheidung des Bundesgerichts wurde in der Schweiz und über die schweizerischen Grenzen hinaus zu einem «Meilenstein» in der Diskussion rund um die fahrlässige Mittäterschaft. ${ }^{23}$

\section{Argumente gegen eine fahrlässige Mittäterschaft und deren Relativierung}

18 Die Lehre deutete den Rolling-StonesFall tendenziell als Anerkennung einer fahrlässigen Mittäterschaft durch das Bundesgericht, obwohl es - nach traditioneller Lehre - eine solche nicht gebe. Zahlreiche Argumente wurden und werden seither gegen die Möglichkeit einer fahrlässigen Mittäterschaft vorgetragen.

\section{Kein gemeinsamer Tatentschluss?}

19 Das Hauptargument, welches gegen eine fahrlässige Mittäterschaft vorgebracht wird, lautet, diese sei nicht möglich, weil Mittäterschaft einen gemeinsamen Tatentschluss voraussetze. Da ein solcher

22 BGE 113 IV 58, 60.

23 Vgl. die zahlreichen Nachweise in Daniel Häring (Fn. 9, 2005), S. 66, Fn. 241. gemeinsamer Tatentschluss bei fahrlässigem Zusammenwirken nicht vorliegen könne, sei auch keine fahrlässige Mittäterschaft möglich. 24

20 Auch das Bundesgericht hat sich in einem obiter dictum in diesem Sinne geäussert. In BGE 126 IV 84 beschäftigte sich das Gericht zwar primär mit der Mittäterschaft bei Verkehrsdelikten und der Frage, ob jemand Täter von Verkehrsdelikten sein könne, der das Fahrzeug nicht selbst lenke. Im Zuge der allgemeinen Erwägungen zur (vorsätzlichen) Mittäterschaft führte das Bundesgericht aus, ohne dass der Fall zu dieser Überlegung Anlass gegeben hätte und ohne Auseinandersetzung mit der eigenen RollingStones-Praxis, die Mittäterschaft würde eine Entscheidung voraussetzen, die zumindest Eventualvorsatz verlange. Folglich sei eine fahrlässige Mittäterschaft nicht denkbar. ${ }^{25}$

21 Dem kann nicht gefolgt werden. Wenn bei fahrlässiger Einzeltäterschaft kein Tatentschluss bzw. Vorsatz gefordert wird, warum soll ein solcher bei fahrlässiger Mittäterschaft vorliegen müssen? Selbstverständlich liegt bei einem gemeinsam ausgeführten Fahrlässigkeitsdelikt kein gemeinsamer Entschluss zu einem Delikt vor, sonst wäre es kein Fahrlässigkeitsdelikt mehr, sondern ein Vorsatzdelikt. Die fahrlässige Mittäterschaft kann aber rein logisch nicht deshalb unmöglich sein, weil Tatbestandselemente der vorsätzlichen Mittäterschaft nicht vorliegen - ja begriffsnotwendig gar nicht vorkommen können. Niemand

24 So u.a. Trechsel/Noll/Pieth (Fn. 8), S. 198 ff.; Andreas Donatsch (Fn. 8, 1989), S. 111; Donatsch/Tag (Fn. 8), S. 370; Bernhard Corboz (Fn. 8), Art. $12 \mathrm{~N} 180$.

25 BGE 126 IV 84, 88 = Pra 2001, Nr. 19, S. 105. 
käme schliesslich auf die Idee zu behaupten, es gäbe keine fahrlässige Einzeltäterschaft, weil vorsätzliche Einzeltäterschaft Vorsatz verlange, und dieser bei fahrlässiger Einzeltäterschaft nicht vorkomme. Gemeinsamkeit bei Fahrlässigkeit darf nicht mit den Kriterien der Gemeinsamkeit beim Vorsatzdelikt (d.h. gemeinsamer Tatentschluss und arbeitsteilige Verwirklichung) definiert werden. Vielmehr muss die fahrlässige Mittäterschaft nach eigenen Tatbestandselementen strukturiert werden, ohne Übernahme der Elemente der vorsätzlichen Mittäterschaft. Dieses Argument gegen eine fahrlässige Mittäterschaft erweist sich somit bereits im Kern als zirkulär. ${ }^{26}$

\section{Keine gesetzliche Grundlage für eine fahrlässige Mittäterschaft?}

Weiter wird gegen eine fahrlässige Mittäterschaft vorgebracht, diese sei mangels gesetzlicher Grundlage nicht möglich. ${ }^{27}$ Allerdings ist auch die vorsätzliche Mittäterschaft nicht gesetzlich geregelt. Trotzdem hat sie sich als strafbarkeitserweiternde Form der Zurechnung, ohne hinreichende gesetzliche Grundlage, in Lehre und Rechtsprechung etabliert und ist heute allgemein anerkannt. Weshalb das Argument der fehlenden gesetzlichen Grundlage ausschliesslich gegen eine fahrlässige Mittäterschaft sprechen soll,

$26 \overline{\text { Ebenso BSK StGB-Riedo (Fn. 7), Art. } 32 \mathrm{~N} \mathrm{21}}$; Riedo/Chvojka (Fn. 11), S. 156 ff.; Harald Maihold, Strafrecht Allgemeiner Teil, 3. A. Basel 2013, S. 279; Simone Kamm, Die fahrlässige Mittäterschaft, Diss. Regensburg 1998, Berlin 1999, S. 107 f.; Franz Riklin, Schweizerisches Strafrecht, Allgemeiner Teil I, Verbrechenslehre, 3. Aufl., Zürich/Basel/Genf 2007, § 18 N 84; sowie Daniel Häring (Fn. 9, 2005), S. 78 ff.; derselbe (Fn. 9, 2008), S. 70 f.

27 Andreas Donatsch (Fn. 8, 2013), Art. 12 N 28; Philippe Spitz (Fn. 8), S. 452; Hans Walder (Fn. 8), S. 57. ist nicht ersichtlich. Auch diese kann sich in Lehre und Rechtsprechung etablieren, ohne dass eine fehlende gesetzliche Grundlage dagegen sprechen würde. ${ }^{28}$

\section{Keine Möglichkeit der Unterschei- dung von Beteiligungsrollen?}

23 Es ist zu lesen, beim Fahrlässigkeitsdelikt sei es nicht möglich, unterschiedliche Beteiligungsformen voneinander abzugrenzen; deshalb sei auch die Konstruktion einer Mittäterschaft beim Fahrlässigkeitsdelikt nicht möglich. ${ }^{29}$

24 Dagegen ist zunächst einzuwenden, dass die Unmöglichkeit der Unterscheidung einer vertikalen Beteiligungsstruktur (d.h. Täterschaft zu Teilnahme) nicht zur Unmöglichkeit der Unterscheidung einer horizontalen Beteiligungsstruktur (d.h. unterschiedliche Formen der Täterschaft) führen kann.30 Darüber hinaus gibt es auch beim Fahrlässigkeitsdelikt durchaus die Möglichkeit, zwischen Täterschaft und Teilnahme zu unterscheiden. Wenn etwa die (fahrlässige) Handlung eines an einem Fahrlässigkeitsdelikt Beteiligten lediglich darauf abzielt, die erfolgsverursachende Fahrlässigkeitshandlung eines anderen Beteiligten zu veranlassen oder zu fördern, erscheint dies als fahrlässige Anstiftung oder Gehilfenschaft bzw. als Anstiftung oder Ge-

28 Daniel Häring (Fn. 9, 2008), S. 71; Riedo/Chvojka (Fn. 11), S. 158 und Harald Maihold (Fn. 26), S. 280.

29 Vgl. etwa Günther Stratenwerth (Fn. 8), § 16 N 49; BSK StGB-Niggli/Maeder (Fn. 7), Art. 12 $\mathrm{N} 134$.

30 So kommen Riedo/Chvojka (Fn. 11), S. 160 ff., auch ohne Unterscheidung vertikaler Beteiligungsstrukturen $\mathrm{zu}$ einem Lösungsansatz, welcher der fahrlässigen Einzeltäterschaft auf horizontaler Ebene eine fahrlässige gemeinsame Täterschaft (Unsorgfaltsgemeinschaft) gegenüberstellt. 
hilfenschaft beim Fahrlässigkeitsdelikt. ${ }^{11}$ Tatsächlich wurde in der Lehre schon mehrfach aufgezeigt, wie auch beim Fahrlässigkeitsdelikt Alleintäter, Mittäter und mittelbare Täter sowie blosse Teilnehmer (Anstifter und Gehilfen) voneinander abgegrenzt werden können. ${ }^{2}$

\section{Ausweitung der Strafbarkeit?}

25 Schliesslich ist durch die Anerkennung einer Mittäterschaft beim Fahrlässigkeitsdelikt auch keine Ausweitung der Strafbarkeit zu befürchten.33 Zum einen werden solche Fälle bereits jetzt regelmässig unter der Rechtsfigur der fahrlässigen Nebentäterschaft bestraft. Zum anderen würde eine konsequente Unterscheidung sämtlicher Beteiligungsformen beim Fahrlässigkeitsdelikt - d.h. inkl. der Anerkennung von fahrlässiger Anstiftung und fahrlässiger Gehilfenschaft vielmehr dazu führen, dass mangels Strafbarkeit von fahrlässiger Teilnahme die strafrechtliche Haftung bei fahrlässigen Handlungen eingegrenzt würde. 34

$3 1 \longdiv { \text { So könnte etwa die sorgfaltswidrige Aufforderung } }$ des Beifahrers, doch mal so richtig «Gas» zu geben, als eine fahrlässige Anstiftung zum Rasen bzw. der dadurch u.U. verursachten Todesfolge eines Passanten betrachtet werden, vgl. Daniel Häring (Fn. 9, 2005), S. 120 Fn. 450.

32 Vgl. etwa BSK StGB-Riedo (Fn. 7), Art. $32 \mathrm{~N} 22$ ff. und N 25 ff.; Daniel Häring (Fn. 9, 2005), S. 113 ff., insb. S. 118 ff., und mit umfassenden Nachweisen auf S. 86, Fn. 317; José Hurtado Pozo, Droit pénal, Partie générale, Genf/Zürich/ Basel 2008, N 1409; Harro Otto, Täterschaft und Teilnahme im Fahrlässigkeitsbereich, Festschrift für Günter Spendel, Berlin/New York 1992, S. $271 \mathrm{ff}$;; Simone Kamm (Fn. 26), S. 108 f.; Joachim Renzikowski, Restriktiver Täterbegriff und fahrlässige Beteiligung, Habil. Tübingen 1997, S. 261 ff., 272 ff., 292 ff., 300 f. und passim. Vgl. dazu auch Harald Maihold (Fn. 26), S. 280.

33 So die Befürchtung z. B. von Hans Walder (Fn. 8), S. 57.

34 Daniel Häring (Fn. 9, 2008), S. 71; vgl. auch BSK StGB-Niggli/Maeder (Fn. 7), Art. 12 N 134.

\section{Fazit}

26 Die gegen eine fahrlässige Mittäterschaft vorgetragenen Argumente überzeugen nicht. Deshalb geht die neuere Lehre auch vermehrt von der Möglichkeit einer Mittäterschaft beim Fahrlässigkeitsdelikt aus oder vertritt zumindest eine differenzierte Auffassung. 35

\section{IV. (Tatbestands-)Elemente einer Mittäterschaft beim Fahrlässigkeitsdelikt}

27 Über die einzelnen Voraussetzungen einer fahrlässigen Mittäterschaft besteht unter den Befürwortern einer solchen Möglichkeit noch kein abschliessender Konsens; immerhin gehen die verschiedenen Ansätze in die ähnliche Richtung.

35 Gunter Arzt, Vorsatz und Fahrlässigkeit, recht 1988, S. 66 ff., 72; BSK StGB-Niggli/Maeder (Fn. 7), Art. 12 N 134; BSK StGB-Forster (Fn. 7), Vor Art. $24 \mathrm{~N} 21$ ff.; BSK StGB-Riedo (Fn. 7), Art. 32 N 20 ff.; Daniel Häring (Fn. 9, 2005), insb. S. 192 ff. und passim; derselbe (Fn. 9, 2008), S. 64 ff.; José Hurtado Pozo (Fn. 32), N 1409; Harald Maihold (Fn. 26), S. 277 ff.; Riedo/Chvojka (Fn. 11), S. 152 ff.; Martin Schubart, Wie entsteht ein Urteil? - Zur Realität der Urteilsfindung, recht 1992, S. 122 ff., S. 126; derselbe, Unteilbarkeit des Strafantrages?, ZStrR 1994, S. 220 ff., S. 223 f.; derselbe, Der Richter zwischen Rationalität und Sensibilität, recht 1995, S. 151 ff., insb. S. 152 und 156; derselbe (Fn. 21), S. 368 ff.; Kurt Seelmann/Christopher Geth, Strafrecht Allgemeiner Teil, 6. A. Basel 2016, S. 180 f. N 501 ff.; differenzierend Bernhard Sträuli (Fn. 12), Intro aux art. 24 à $27 \mathrm{~N} 115 \mathrm{ff}$. In Deutschland ist die Möglichkeit einer Mittäterschaft beim Fahrlässigkeitsdelikt bereits zu grossen Teilen anerkannt, vgl. statt vieler Kamm (Fn. 26), S. 195 ff., 214 ff. und passim; Harro Otto, Mittäterschaft beim Fahrlässigkeitsdelikt, Jura 1990, S. 47 ff.; derselbe (Fn. 32), S. 271 ff.; Joachim Renzikowski (Fn. 32), S. 282 ff.; Claus Roxin, Täterschaft und Tatherrschaft, 9. Aufl., Habil. Göttingen 1963, Berlin/Boston 2015, S. 770 ff., mit vielen weiteren Nachweisen insb. in Fn. 895; Bettina Weisser (Fn. 13), S. 230 ff. 
Nachfolgend sei ein möglicher Ansatz präsentiert. ${ }^{6} 6$

Vorab sei aber noch eine begriffliche Präzisierung erlaubt: Der verbreitete Begriff «fahrlässige Mittäterschaft» ist irreführend. Nicht die Mittäterschaft wird fahrlässig begangen, sondern ein Fahrlässigkeitsdelikt wird durch mehrere Personen ausgeführt. Richtig ist also die Frage, ob es eine Mittäterschaft sowohl beim Vorsatz- als auch beim Fahrlässigkeitsdelikt gibt. 37 Anstelle einer fahrlässigen Mittäterschaft wäre es deshalb treffender, von einer Mittäterschaft beim Fahrlässigkeitsdelikt zu sprechen.

Mittäterschaft bedeutet gemeinsame Täterschaft. Deshalb muss es zunächst einmal gelingen, einen hinreichend präzisen Begriff fahrlässiger «Gemeinsamkeit» zu bilden. $3^{8}$ Da sich die Gemeinsamkeit beim Fahrlässigkeitsdelikt begriffsnotwendig nicht auf die Verwirklichung des gesetzlichen Tatbestands beziehen kann, muss sie sich auf die natürliche(n) Handlung(en) beziehen, die dem sorgfaltswidrigen Verhalten zugrunde liegen bzw. deren sorgfaltswidrige Ausführung den Fahrlässigkeitserfolg herbeigeführt hat. Die gemeinsame Erfolgsherbeiführung definiert sich beim Fahr-

36

Dieser Ansatz wurde bereits dargestellt in Daniel Häring (Fn. 9, 2008), S. 72 f. Vgl. zum Stand der Diskussion Daniel Häring (Fn. 9, 2005), S. $164 \mathrm{ff}$. Als mögliche Alternativen zur vorgeschlagenen Lösung insb. Riedo/Chvojka (Fn. 11), S. 152 ff.; sowie die in Fn. 35 genannten Autoren.

37 René Bloy, Rezension zum Werk «Die Fahrlässige Mittäterschaft» von Simone Kamm, GA 2000, S. 392 ff., S. 395; Daniel Häring (Fn. 9, 2005), S. 193. Da der Begriff «fahrlässige Mittäterschaft» bereits weit verbreitet ist, wird er in der vorliegenden Untersuchung auch vereinzelt verwendet.

38 So auch die Ausgangslage für Claus Roxin (Fn. 35), S. 771. lässigkeitsdelikt somit über die gemeinsame Vornahme der zum Fahrlässigkeitserfolg führenden natürlichen Handlung(en). 39

30 Zum Vergleich: Gemeinschaftskonstituierende Elemente der vorsätzlichen Mittäterschaft sind subjektiv der gemeinsame Tatentschluss, objektiv die arbeitsteilige Ausführung der Gesamttat bzw. Mittätertat. Diese Überlegungen können auch beim Fahrlässigkeitsdelikt analog Anwendung finden: Auch hier bedarf es eines bewussten Zusammenwirkens der Beteiligten bei der Ausführung der natürlichen Handlung(en), welche sich als Ursache des Fahrlässigkeitserfolgs zeigen. Die zwischen den am Fahrlässigkeitsdelikt Beteiligten notwendige Gemeinsamkeit besteht subjektiv aus dem gemeinsamen Entschluss zur arbeitsteiligen Ausführung der zum Fahrlässigkeitserfolg führenden Handlung(en). Man kann von einem gemeinsamen Handlungsentschluss sprechen. Objektiv gemeinschaftskonstituierendes Element ist beim Fahrlässigkeitsdelikt das arbeitsteilige Zusammenwirken bei der Ausführung derjenigen natürlichen Handlung(en), die sich objektiv als Ursache des eingetretenen Fahrlässigkeitserfolgs zeigen. Damit ist die Gemeinsamkeit beim Fahrlässigkeitsdelikt hinreichend definiert. 40

39 BSK StGB-Riedo (Fn. 7), Art. 32 N. 22; Daniel Häring (Fn. 9, 2005), S. 196 ff.; derselbe (Fn. 9, 2008), S. 72; Seelmann/Geth (Fn. 35), S. 181 N 502; Harald Maihold (Fn. 26), S. 278.

40 Vgl. dazu ausführlich Daniel Häring (Fn. 9, 2005), S. 196 ff.; derselbe (Fn. 9, 2008), S. 72; ähnlich BSK StGB-Riedo (Fn. 7), Art. $32 \mathrm{~N}$ 22; Seelmann/Geth (Fn. 35), S. 181 N 502; Harald Maihold (Fn. 26), S. 278; José Hurtado Pozo (Fn. 32), N 1409; im Ergebnis bereits BGE 113 IV 58. 
31 Weiter ist zu fordern, dass jeder Mittäter täterschaftlich handelt. Das Bestehen einer subjektiven und objektiven Gemeinsamkeit beim Fahrlässigkeitsdelikt, welche es rechtfertigt, von gemeinsamer Vornahme der zum Fahrlässigkeitserfolg führenden Handlung(en) zu sprechen, begründet für sich noch keine strafrechtliche Verantwortlichkeit aller Beteiligten für die durch diese Handlung(en) herbeigeführten Erfolge. Vielmehr ist zu verlangen, dass jeder Mittäter das Täterkriterium erfüllt. Die am Fahrlässigkeitsdelikt Beteiligten müssen somit gestützt auf den gemeinsamen Handlungsentschluss einen individuellen Einzelbeitrag bei dessen Umsetzung, d.h. bei der arbeitsteiligen Ausführung der Erfolg verursachenden Handlung(en) erbringen. Dieser individuelle Handlungsbeitrag muss für das ganze Projekt wesentlich sein und eine individuelle Sorgfaltspflichtverletzung der jeweils handelnden Person darstellen. ${ }^{41}$ In Anlehnung an die gemeinsame (funktionale) Tatherrschaft beim Vorsatzdelikt könnte man beim Fahrlässigkeitsdelikt von gemeinsamer (funktionaler) Handlungsherrschaft sprechen.

Die Mittäterschaft führt sowohl beim Vorsatz- als auch beim Fahrlässigkeitsdelikt zu einer wechselseitigen Zurechnung der Handlungsbeiträge der Beteiligten. Deshalb ist bei der Prüfung der (natürlichen) Kausalität danach zu fragen, ob die Handlungen aller Beteiligten (und nicht von jedem einzeln) den Taterfolg mit an Sicherheit grenzender Wahrscheinlichkeit herbeigeführt haben. Nach Feststellung der Kausalität ist in einem weiteren
Schritt zu prüfen, inwieweit dem einzelnen Beteiligten der gemeinsam herbeigeführte Fahrlässigkeitserfolg individuell zugerechnet werden kann. Dabei ist zu verlangen, dass der Einzelne durch seine pflichtwidrig unvorsichtig ausgeführte individuelle Einzelhandlung ein tatbestandsrelevantes unerlaubtes Risiko geschaffen hat, welches sich, gemeinsam mit den durch die übrigen Beteiligten geschaffenen unerlaubten Risiken, in generell und individuell voraussehbarer und vermeidbarer Weise im tatsächlich eingetretenen Fahrlässigkeitserfolg (mit-) realisiert hat. 42

33 Somit ergeben sich folgende Tatbestandselemente einer Mittäterschaft beim Fahrlässigkeitsdelikt im Überblick:43 (i) Die Beteiligten fassen einen gemeinsamen Handlungsentschluss zur arbeitsteiligen Vornahme der natürlichen Handlung(en), deren sorgfaltswidrige Ausführung zum Fahrlässigkeitserfolg geführt haben; (ii) sie führen den gemeinsamen Handlungsentschluss in arbeitsteiligem Zusammenwirken aus; (iii) dabei erbringt jeder Einzelne einen wesentlichen Einzelbeitrag, der sich für ihn als individuelle Sorgfaltspflichtverletzung erweist; (iv) die Handlungen aller Beteiligten müssen den Fahrlässigkeitserfolg gemeinsam (natürlich) kausal verursacht haben, und (v) der Fahrlässigkeitserfolg muss für jeden Beteiligten vorhersehbar und vermeidbar sowie jedem Beteiligten individuell objektiv zurechenbar sein.
41 Daniel Häring (Fn. 9, 2008), S. 72 sowie ausführlich derselbe (Fn. 9, 2005), S. 242 ff. D.h. die Annahme von Mittäterschaft entbindet nicht vom Erfordernis einer individuellen Sorgfaltspflichtverletzung jedes Beteiligten.
42 Daniel Häring (Fn. 9, 2008), S. 73 sowie ausführlich derselbe (Fn. 9, 2005), S. 276 ff. und $281 \mathrm{ff}$.

43 Vgl. bereits Daniel Häring (Fn. 9, 2008), S. 73. 


\section{Das Urteil des Bundesgerichts im Lichte der Mittäterschaft beim Fahrlässigkeitsdelikt}

\section{Einleitung}

34 Voranstehend wurde das wiederkehrende Problem dargestellt und aufgezeigt, dass die klassischen Lösungsansätze mit Ungereimtheiten behaftet sind. Es wurde herausgearbeitet, dass die Gründe, welche gemeinhin gegen eine Mittäterschaft beim Fahrlässigkeitsdelikt vorgetragen werden, nicht überzeugen. Schliesslich wurde aufgezeigt, wie die Tatbestandsmerkmale einer solchen Rechtsfigur definiert werden können.

Zum Abschluss der vorliegenden Untersuchung soll nun der Entscheid des Bundesgerichts im Ausgangsfall (Raketenfall) dargestellt und im Lichte der Mittäterschaft beim Fahrlässigkeitsdelikt analysiert werden.

\section{Die vorinstanzlichen Urteile}

36 In den vorinstanzlichen Verfahren gaben sich jeweils Schuld- und Freisprüche die Hand:

37 Zunächst wurden die beiden Beschuldigten mit separaten Strafbefehlen und einer auf den Rolling-Stones-Entscheid gestützten Begründung wegen fahrlässiger Verursachung einer Feuersbrunst sowie Widerhandlung gegen das Brandschutzgesetz bestraft. 44

38 Im nachfolgenden erstinstanzlichen Verfahren kam der Präsident des Bezirksgerichts Laufenburg zum Schluss, dass es an der individuellen Zurechenbarkeit

44 Urteil des Bundesgerichts 6B_360/2016, 6B_361/2016 vom 1. Juni 2017, Sachverhalt A. fehle. Der Hinweis auf den RollingStones-Entscheid ändere daran nichts, weil im konkreten Fall kein gemeinsamer Beschluss einer sorgfaltswidrigen Handlung vorliege. Vielmehr sei «nur» von einem nicht abgesprochenen Fehlverhalten zweier gedankenloser Männer auszugehen. Er sprach beide Angeklagten frei. 45

39 Das Obergericht des Kantons Aargau sprach A und B der fahrlässigen Verursachung einer Feuersbrunst (Art. 222 Abs. 1 StGB) schuldig. Es vertrat die Meinung, die beiden Beschuldigten hätten gemeinsam beschlossen, vier gleichartige Feuerwerksraketen arbeitsteilig zu starten. Es sei davon auszugehen, dass sie jeweils für sich und ohne vorgängige Absprache beschlossen, die Raketen in der zwar gefährlichen, aber nicht unüblichen Weise durch jeweiliges In-denBoden-Stecken auf dem Rasenplatz direkt vor der Wohnung des ersten Beschwerdeführers mit nur wenigen Metern Abstand in unmittelbarer Nähe zum in Brand geratenen Balkon abzufeuern. Es sei allgemein bekannt sowie erkennbar und ergebe sich aus Sicherheitsempfehlungen, dass ein ausreichender Sicherheitsabstand nicht nur zu Personen, sondern auch zu Gebäuden eingehalten werden müsse. Nach der Website der Beratungsstelle für Brandverhütung (BFB) sei je nach Grösse des Feuerwerkskörpers ein Sicherheitsabstand von mindestens 40m zu Gebäuden, Getreidefeldern oder Waldrändern erforderlich. Aufgrund des Augenscheins sei klar, dass der auch nur für ein halbwegs sicheres Abfeuern erforderliche Minimalabstand nicht eingehalten wurde und jede von der

45 Urteil des Bundesgerichts 6B_36o/2016, 6B_361/2016 vom 1. Juni 2017, Sachverhalt A und B sowie E. $4 \cdot 3$. 
Senkrechten abweichende Flugbahn einen gefährlichen Irrflug darstellte. Der Brand sei direkte Folge der gemeinsam beschlossenen und vorgenommenen Gesamthandlung, die als sorgfaltswidrig zu bezeichnen sei. 46

\section{Das Urteil des Bundesgerichts in der Sache}

40 Das Bundesgericht entschied den Fall nicht primär aufgrund rechtlicher, sondern insbesondere tatsächlicher Umstände. Es hielt gestützt auf den vom Obergericht verbindlich festgestellten Sachverhalt fest, dass die Beschuldigten zwar beschlossen hätten, arbeitsteilig vier Raketen zu starten. Es habe jedoch nicht festgestellt werden können, dass sie auch beschlossen hätten, dies durch ein unsorgfältiges In-den-Boden-Stecken der Raketen zu tun. Dies sei jedoch ursächlich gewesen für den schadensverursachenden Irrläufer. Vielmehr hätten die Beteiligten jeweils für sich, d.h. ohne vorgängige Absprache, die Raketen in dieser nicht unüblichen Weise abgefeuert. Aus dieser Beweisführung folge, dass die beiden Beschuldigten nicht beschlossen hätten, ein Risiko einzugehen; sie hätten einzig beschlossen, Raketen $\mathrm{zu}$ starten. Die (pflichtwidrige) Art und Weise des Startens bestimmte hingegen jeder für sich, d.h. ohne vorgängige Absprache.47 In Bezug auf das eigentlich sorgfaltswidrige Element des «falschen» bzw. «gefährlichen» Abfeuerns liege keine gemeinsame Absprache vor, mit anderen Worten sei der gemeinsame Beschluss einer sorgfaltswidrigen Handlung

46 Urteil des Bundesgerichts 6B_360/2016, 6B_361/2016 vom 1. Juni 2017, Sachverhalt B und E. 3.2.

47 Urteil des Bundesgerichts 6B_360/2016, 6B_361/2016 vom 1. Juni 2017, E. 4.5 und E. 4.6. nicht bewiesen.$^{48}$ Bei einer solchen Sachverhaltskonstellation, so das Bundesgericht, dürfe insbesondere der RollingStones-Fall nicht als Vergleichsfall dienen:

«Mithin fehlt es an den Voraussetzungen der Annahme einer Gesamthandlung im Sinne von BGE 113 IV 58 [...]. Das Bundesgericht hat deshalb weder auf BGE 113 IV 58 einzugehen noch sich mit der Kausalitätsfrage auseinanderzusetzen.»49

41 Diese Beweislosigkeit, so das Bundesgericht weiter, könne nicht über eine wie auch immer begründete Mittäterschaft substituiert werden. Die Rechtsfigur der Mittäterschaft führe lediglich zu einer Beweiserleichterung hinsichtlich des individuellen Nachweises von Tatbeiträgen, keineswegs aber hinsichtlich des Tatentschlusses.50 Daraus folgert das Bundesgericht:

«Auf die Frage, ob und inwiefern sie [Anm: die beiden Beschuldigten] sich auch 'gemeinsam' sorgfaltswidrig verhalten könnten, ist nicht einzugehen. Denn klar ist, dass die Komponente eines sorgfaltswidrigen gemeinsamen Unternehmens [...] in subjektiver Hinsicht in jeder Eventualität erst nachgewiesen werden müsste.» 51

48 Urteil des Bundesgerichts 6B_360/2016, 6B_361/2016 vom 1. Juni 2017, E. 4.9.

49 Urteil des Bundesgerichts 6B_360/2016, 6B_361/2016 vom 1. Juni 2017, E. 4.9.

50 Urteil des Bundesgerichts 6B_360/2016, 6B_361/2016 vom 1. Juni 2017, E. 4.10.

51 Urteil des Bundesgerichts 6B_360/2016, 6B_361/2016 vom 1. Juni 2017, E. 4.10. 
Deshalb kommt das Bundesgericht zum Schluss, dass sich wegen des nur mangelhaft durchgeführten Beweisverfahrens nicht mehr eruieren lasse, welcher der beiden Beschwerdeführer die brandauslösende Rakete gezündet hatte. Insbesondere sei auch deren individuellkonkretes Vorgehen und der diesbezüglich rechtserhebliche subjektive Sachverhalt nicht mehr zu eruieren. Weil dies zu einem prozessualen Leerlauf führen würde, sei davon abzusehen, die Sache nach Art. 112 Abs. 1 lit. b BGG52 an die Vorinstanz zurückzuweisen. Vielmehr seien die Beschwerden gutzuheissen, die vorinstanzlichen Urteile aufzuheben und beide Beteiligten freizusprechen. 53

\section{Analyse des Urteils mit Blick auf die fahrlässige Mittäterschaft}

43 Auch wenn das Bundesgericht die Frage nach einer Mittäterschaft beim Fahrlässigkeitsdelikt letztlich offen lässt, sind im Lichte des Entscheids doch folgende für die Diskussion wichtigen Punkte hervorzuheben.

\section{a) Meinungsstand der Lehre}

44 Das Bundesgericht schreitet im Urteil die schweizerischen Lehrmeinungen zur fahrlässigen Mittäterschaft und zum Rolling-Stones-Entscheid ab; es kommt zum Schluss, dass die Konzeption einer fahr-

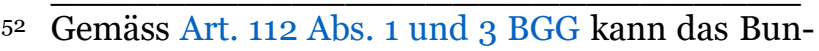
desgericht Entscheide, welche u.a. die massgebenden Gründe tatsächlicher und rechtlicher Art nicht hinreichend beschreiben, zur Verbesserung an die Vorinstanz zurückweisen.

53 Urteil des Bundesgerichts 6B_360/2016, 6B_361/2016 vom 1. Juni 2017, E. 4.11 und E. 5; ausgehend von den Feststellungen des Bundesgerichts wäre in diesem Fall ein Direktfreispruch, der leider selten vorkommt, sinnvoller gewesen. lässigen Mittäterschaft von der wohl herrschenden Lehre abgelehnt werde. 54

45 Das Bundesgericht anerkennt im Urteil immerhin, dass es die Lehre zur Mittäterschaft beim Fahrlässigkeitsdelikt nicht erschöpfend darstellt.55 Dies ist grundsätzlich nicht zu beanstanden. Störend ist vor diesem Hintergrund jedoch die Feststellung, dass eine solche Rechtsfigur von der herrschenden Lehre abgelehnt werde. Tatsächlich wurde die eine Mittäterschaft beim Fahrlässigkeitsdelikt befürwortende Lehre durch das Bundesgericht mit Ausnahme von José Hurtado Pozo samt und sonders überhaupt nicht erwähnt. Richtig ist, dass in der Schweiz neben Hurtado Pozo unter anderem Martin Schubart, Kurt Seelmann/ Christopher Geth, Christoph Riedo, Harald Maihold sowie der Autor dieser Untersuchung eine Mittäterschaft beim Fahrlässigkeitsdelikt ausdrücklich befürworten; weitere Autoren lehnen diese immerhin nicht mehr kategorisch ab. ${ }^{6}$ Richtig ist auch, dass in Deutschland die fahrlässige Mittäterschaft bereits als überwiegende Lehre angesehen werden kann. ${ }^{77}$ Somit darf bezweifelt werden, ob die Feststellung des Bundesgerichts zur heute herrschenden Lehre in dieser Deutlichkeit richtig ist. Vielmehr wird hier die These vertreten, dass heute ein grosser - wenn nicht sogar der überwiegende - Teil der Lehre zumindest eine differenzierte Auffassung vertritt, und dass das neuere Schrifttum, wenn es sich

54 Urteil des Bundesgerichts 6B_360/2016, 6B_361/2016 vom 1. Juni 2017, E. 4.7.

55 So ausdrücklich das Bundesgericht in E. 4.7. des Entscheids.

56 Vgl. die detaillierten Nachweise vorne in Fn. 35.

57 Claus Roxin (Fn. 35), S. 770 ff., insb. mit den Nachweisen in Fn. 895; so auch BSK StGB-Riedo (Fn. 7), Art. 32 N 22. 
denn mit der Sache etwas intensiver beschäftigt, sich zu grössten Teilen in Richtung Anerkennung einer Mittäterschaft beim Fahrlässigkeitsdelikt entwickelt.

\section{b) Frage der Mittäterschaft beim Fahrlässigkeitsdelikt noch nicht höchstrichterlich entschieden}

46 Das Bundesgericht untersucht im Urteil seine bisherige Praxis. Es zeigt auf, dass es in früheren Fällen zwar Berührungspunkte mit einem Zusammenwirken mehrerer Beteiligter beim Fahrlässigkeitsdelikt gegeben hat. Das Bundesgericht stellt jedoch zumindest implizit klar, dass die Frage nach der fahrlässigen Mittäterschaft bisher noch nicht abschliessend höchstrichterlich entschieden ist. 58

Dies ist insbesondere vor dem Hintergrund des vorne bereits erwähnten obiter dictums in BGE 126 IV 84, 88 wichtig. Die dortige Nebenbemerkung des Bundesgerichts, wonach eine Mittäterschaft beim Fahrlässigkeitsdelikt deshalb nicht möglich sei, weil es bei der Fahrlässigkeit keinen gemeinsamen Tatentschluss gebe,59 ist für das Bundesgericht nun offenbar nicht mehr zentral. Das Bundesgericht stellt zu Recht klar, dass die damalige Aussage sich auf den vorsätzlichen Mittäterschaftsbegriff stützte und deshalb gerade nicht auf die Fahrlässigkeitstat übertragen werden kann. ${ }^{60}$

58 Urteil des Bundesgerichts 6B 360/2016, 6B_361/2016 vom 1. Juni 2017, E. 4.8 und E. 4.9.

59 BGE 126 IV 84, 88 = Pra 2001, Nr. 19, S. 105.

60 Urteil des Bundesgerichts 6B_360/2016, 6B_361/2016 vom 1. Juni 2017, E. 4.8

\section{c) Absage an das Argument des angeblich fehlenden Tatentschlusses}

48 Neben der gleich hiervor dargestellten Feststellung durch das Bundesgericht, wonach das obiter dictum in BGE 126 IV 84, 88, letztlich irrelevant sei, weil sich die Schlussfolgerung auf den vorsätzlichen Mittäterschaftsbegriff bezieht, rückt das Bundesgericht die Unterschiede zwischen Vorsatz und Fahrlässigkeit bei der Mittäterschaft auch an anderer Stelle im Entscheid nochmals ins Zentrum. Das Bundesgericht stellt klar, dass die vorsätzliche Mittäterschaft wissentlich und willentlich die gemeinsame Begehung einer Straftat bezweckt, die folglich nur vorsätzlich begangen werden kann. Fahrlässigkeitstäter verhalten sich definitionsgemäss aber gerade nicht so, weshalb - dies ergibt sich aus den Erwägungen des Bundesgerichts zumindest implizit - ein «gemeinsam» sorgfaltswidriges Verhalten nicht über einen gemeinsamem Tatentschluss definiert werden dürfe; vielmehr müssen die subjektiven Komponenten eines sorgfaltswidrigen gemeinsamen Unternehmens anders definiert werden. ${ }^{61}$

49 Mit diesen Ausführungen des Bundesgerichts dürfte dem Hauptargument gegen eine Mittäterschaft beim Fahrlässigkeitsdelikt, diese gebe es nicht, weil es keinen gemeinsamen Tatentschluss gebe, ein weiterer Stein aus dem ohnehin schon brüchigen Fundament gezogen worden sein. Nochmals: Die fahrlässige Mittäterschaft ist nicht deshalb unmöglich, weil ein Tatbestandselement der vorsätzlichen Mittäterschaft darin nicht vorkommt. ${ }^{62}$

61 Urteil des Bundesgerichts 6B_360/2016, 6B_361/2016 vom 1. Juni 2017, E. 4.10.

62 Vgl. dazu umfassend vorne III. 1. 


\section{d) Absage an die Mitverur- sachungstheorie bzw. Nebentäterschaft}

Das Bundesgericht zitiert im Ausgangsentscheid aus eigenen früheren Urteilen mit Verweisen auf die Lehrmeinung Günter Stratenwerths. In diesen Entscheiden wurde ausgeführt, dass bei der Beteiligung mehrerer an einer Fahrlässigkeitstat mangels subjektiver deliktischer Verbindung nur Nebentäterschaft möglich sei, wenn die eigene Handlung des jeweiligen Täters irgendwie zum Risiko des Erfolgseintritts beitragen würde. Das Bundesgericht stützt diese Idee der Nebentäterschaft bei gemeinsamen Handlungen allerdings nicht (mehr):

«Nach dieser Argumentation ist lediglich Nebentäterschaft [...] möglich. Von Nebentäterschaft wird indessen gesprochen, wenn mehrere Täter unabhängig voneinander denselben Tatbestand verwirklichen [...]. Das aber ist eine andere Konstellation als jene in der vorliegenden Fragestellung.»63

51 Es ist erfreulich, wie klar sich das Bundesgericht zu dieser Begriffsverwirrung äussert: Wenn mehrere Täter gemeinsam ein Fahrlässigkeitsdelikt begehen, ist für die Konzeption der Nebentäterschaft kein Raum.

\section{e) Inhalt des gemeinsamen Handlungsentschlusses} Dreh- und Angelpunkt des Bundesgerichtsurteils ist der Inhalt des subjektiv verbindenden Elements der Mittäter an einem Fahrlässigkeitsdelikt, des Hand-

63 Urteil des Bundesgerichts 6B_360/2016, 6B_361/2016 vom 1. Juni 2017, E. 4.8. lungsentschlusses. Das Bundesgericht verneinte eine gemeinsame Verantwortlichkeit mit dem Argument, die Täter hätten lediglich den nicht grundsätzlich pflichtwidrigen Entschluss gefasst, gemeinsam Raketen zu zünden. Sie hätten hingegen keinen gemeinsamen Entschluss gehabt, dieses Vorhaben risikoreich durchzuführen (insb. durch falsches In-den-Boden-Stecken). ${ }^{64}$ Somit ist zu prüfen, ob ein gemeinsamer Handlungsentschluss, der den ersten Anknüpfungspunkt für eine fahrlässige Mittäterschaft bildet (oder, neutraler, einer Gesamthandlung nach dem Rolling-StonesFall), sich zwingend auch auf die unsorgfältige, pflichtwidrige Handlungsausführung beziehen muss.

53 Dies ist zu verneinen. Der gemeinsame Handlungsentschluss als Kriterium der subjektiven Gemeinsamkeit der Mittäter muss sich allein auf die aussertatbestandliche Handlung beziehen, die sich im Nachgang als unsorgfältig ausgeführt darstellt. Der Entschluss selbst muss weder sorgfaltswidrig sein, noch sich auf die unsorgfältige Handlungsausführung beziehen, noch sonst einen deliktischen Sinnbezug aufweisen. Es ist die dem Beschluss normalerweise zeitlich folgende pflichtwidrige Umsetzung, welche den beim Fahrlässigkeitsdelikt pönalisierten Deliktserfolg herbeiführt. Die unsorgfältige Handlungsvornahme, die vorhersehbar und vermeidbar den Deliktserfolg hervorruft, ist strafrechtlicher Anknüpfungspunkt der Fahrlässigkeitstat, nicht der subjektive Wille, diese Handlung auszuführen. Mit anderen Worten ist die Zielsetzung der fahrlässigen Handlung und zwar unabhängig von Einzel- oder

64 Urteil des Bundesgerichts 6B_36o/2016, 6B_361/2016 vom 1. Juni 2017, E. 4.9. 
Mittäterschaft -, nicht deren strafbewehrter Anknüpfungspunkt. 65

54 Deshalb werden auch bewusste und unbewusste Fahrlässigkeit bestraft: Bei erstem ist dem Täter bewusst, dass seine Handlung unsorgfältig ist und er weiss, dass ein Deliktserfolg eintreten könnte; bei der unbewussten Fahrlässigkeit ist dies gerade nicht der Fall, der Täter bedenkt die Gefahr der Tatbestandsverwirklichung nicht einmal. ${ }^{66} \mathrm{Ob}$ der Täter einen subjektiv-finalen Bezug zur Pflichtwidrigkeit der Handlung hat, ist für die grundsätzliche Begründung der Fahrlässigkeitsstrafe also irrelevant.

Somit kann es auch zur Begründung von subjektiver Gemeinsamkeit beim Fahrlässigkeitsdelikt nicht darauf ankommen, ob sich der Beschluss der Handlung nun auch darauf bezieht, diese sorgfältig oder unsorgfältig auszuführen. Der gemeinsame Handlungsentschluss, welcher die subjektive Verbindung der Mittäter am Fahrlässigkeitsdelikt begründet, bezieht sich auf die bewusste (finale) Vornahme der (Gesamt-)Handlung, nicht aber auf den strafrechtlich relevanten Umstand, dass diese unvorsichtig ausgeführt wird und deshalb einen Fahrlässigkeitserfolg herbeiführt. ${ }^{67}$

65 Vgl. ausführlich Daniel Häring (Fn. 9, 2005), S. 198 f., mit vielen weiteren Nachweisen in Fn. 764 und 765 , S. 218 f. und S. 225 ff.

66 So bereits Art. 12 Abs. 3 StGB, wonach fahrlässig ein Verbrechen auch begeht, wer die Folge seines Verhaltens «nicht bedenkt»; vgl. auch BSK StGBNiggli/Maeder (Fn. 7), Art. 12 N 85.

67 Daniel Häring (Fn. 9, 2005), S. 198 f., S. 218 f. und S. 225 ff.; auch Seelmann/Geth (Fn. 35), S. 181 N 502 und Harald Maihold (Fn. 26), S. 279, sprechen nicht davon, dass der Handlungsentschluss einen deliktischen Sinnbezug haben bzw. sich auf die Unsorgfalt beziehen muss, vielmehr beziehe sich dieser auf «jene natürliche Handlung, die dem sorgfaltswidrigen Verhalten zugrunde liegt» (Seelmann/Geth) bzw.
56 Zur Vermeidung allfälliger Konfusionen sei hervorgehoben, dass das Kriterium des gemeinsamen Handlungsentschlusses nichts mit der Vorhersehbarkeit des Taterfolgs zu tun hat. Das Kriterium der Voraussehbarkeit verlangt, dass die pflichtwidrig unvorsichtige Handlung und der Erfolg nicht nur objektiv durch die natürliche Kausalität, sondern auch subjektiv insoweit miteinander verknüpft sein müssen, dass der Täter - ex ante zumindest hätte erkennen können, dass und auf welche Weise (Kausalverlauf) der Fahrlässigkeitserfolg tatsächlich eintreten werde. ${ }^{68}$ Dies muss auch bei fahrlässiger Mittäterschaft gegeben sein. ${ }^{69} \mathrm{Al}-$ lerdings ist das Kriterium ein rechtliches Element der Eingrenzung der Verantwortlichkeit, während der gemeinsame Handlungsentschluss ein tatsächliches Element der Begründung von Gemeinsamkeit darstellt. Die mittäterschaftliche Gemeinsamkeit bezieht sich nicht auf die objektive Zurechnung. Deshalb muss neben dem gemeinsamen Handlungsentschluss für jeden Mittäter einzeln der Fahrlässigkeitserfolg und der dazu führende Geschehensablauf in den wesentlichen Zügen vorhersehbar sein. ${ }^{70}$

auf ein «aussertatbestandliches Handlungsziel» (Harald Maihold); ähnlich auch Alexander Schaal, Strafrechtliche Verantwortlichkeit bei Gremienentscheidungen in Unternehmen, Diss. Tübingen 1999, Berlin 2001, S. 240 ff., der darauf hinweist, dass strafrechtlich relevante «Handlungsprojekte» durchaus auch neutrale Ziele haben können. Allenfalls anderer Meinung, wenn auch nicht explizit, José Hurtado Pozo (Fn. 32), $\mathrm{N}$ 1409: «[...] les auteurs pouvaient et devaient connaître le caractère dangereux de leur comportement»; doch kann sich diese Aussage allenfalls auch auf das Kriterium der Vorhersehbarkeit beziehen.

68 Zur Voraussehbarkeit beim Fahrlässigkeitsdelikt (Einzeltäterschaft) vgl. etwa BGE 130 IV 7, 10; BGE 129 IV 282, 284; BGE 118 IV 130, 134.

69 Vgl. dazu vorne IV. sowie ausführlich Daniel Häring (Fn. 9, 2005), S. 283 ff.

70 Daniel Häring (Fn. 9, 2005), S. 281 f. und S. 283. 
Nach der hier vertretenen Ansicht wäre es also richtig gewesen, den gemeinsamen Handlungsentschluss im Ausgangsfall anzunehmen, auch wenn sich dieser nicht auf die gefährliche Art und Weise des Raketenabfeuerns bezogen hat. Der subjektiv verbindende Teil für die Annahme einer Gesamthandlung lag somit vor. Erst unter dem Kriterium der individuellen Vorhersehbarkeit wäre für jeden Täter einzeln zu prüfen gewesen, ob er im ganz konkreten Fall die Gefährlichkeit des Gesamtprojekts hätte erkennen und den Taterfolg hätte vorhersehen können.

Schliesslich muss auch folgende Eventualüberlegung berücksichtigt werden. Selbst wenn man der Auffassung folgen wollte, dass sich der gemeinsame Handlungsentschluss explizit auch auf die Unsorgfalt der (nachfolgenden) Handlungsausführung beziehen muss, wären immerhin die Grundsätze des konkludenten Handelns in Betracht zu ziehen. Es ist allgemein anerkannt, dass ein Tatentschluss (bei vorsätzlicher Mittäterschaft) - und somit konsequenterweise auch ein Handlungsentschluss (bei fahrlässiger Mittäterschaft) - durch konkludentes Handeln entstehen kann. ${ }^{71}$ Im konkreten Fall etwa hätte es durchaus ausreichen können, dass nach dem explizit gefassten Beschluss, Raketen im Freien (sorgfältig) abzufeuern, das Element der Pflichtwidrigkeit nachträglich zum Beschluss hinzutrat bzw. nachträglich vom Beschluss umfasst wurde, als die Raketen dann von beiden Beteiligten tatsächlich unsorgfältig (durch In-den-Boden-Stecken) abgefeuert wurden. Der ursprünglich rechtmässige Beschluss des Raktenabfeuerns

71 Vgl. statt vieler der hier diskutierte Entscheid Urteil des Bundesgerichts 6B_360/2016, 6B_361/2016 vom 1. Juni 2017, E. 4.10. wäre dann durch die tatsächlich unsorgfältige Vornahme der Handlung via konkludentes Handeln zu einem gemeinsamen Unsorgfaltsbeschluss geworden, und das subjektive Element der fahrlässigen Mittäterschaft hätte bejaht werden können. Das Bundesgericht setzt sich mit dieser Möglichkeit im Ausgangsfall nicht explizit auseinander. Generell dürfte aber davon ausgegangen werden, dass ein Beschluss zu pflichtgemässem Handeln spätestens bei der pflichtwidrigen Handlungsvornahme konkludent zu einem Beschluss pflichtwidrigen Handelns wird. ${ }^{2}$

\section{Fazit}

59 Der durch das Bundesgericht beurteilte Ausgangsfall hätte das Potenzial gehabt, die Rechtsfigur einer fahrlässigen Mittäterschaft bzw. einer Mittäterschaft beim Fahrlässigkeitsdelikt zu etablieren. Dies entweder dann, wenn anerkannt wird, dass sich der gemeinsame Handlungsentschluss als subjektives Element der Gemeinsamkeit nicht zwingend auf die Sorgfaltswidrigkeit der Handlung beziehen muss, sondern nur auf die gemeinsame Handlungsvornahme als solche. Oder wenn berücksichtigt wird, dass ein Beschluss von pflichtgemässem Handeln spätestens bei der pflichtwidrigen Handlungsvornahme konkludent zu einem Beschluss von pflichtwidrigem Handeln wird.

6o Immerhin hat das Bundesgericht mit seinem Entscheid einige wichtige Weichen gestellt:

72 Vgl. zum Handlungsentschluss bei fahrlässiger Mittäterschaft durch konkludentes Handeln $D a$ niel Häring (Fn. 9, 2005), S. 221 f. 
1. Zunächst wird einmal mehr evident, dass es Fälle gibt, welche nur mit der Figur einer Mittäterschaft beim Fahrlässigkeitsdelikt befriedigend gelöst werden können.

2. Die Würfel für oder gegen eine fahrlässige Mittäterschaft sind weder in Lehre noch Rechtsprechung bereits in eine klare Richtung gefallen.

3. Es spricht nicht gegen die fahrlässige Mittäterschaft, dass darin ein Element des Vorsatzdelikts nicht vorkommt.

4. Das Konzept der Nebentäterschaft betrifft andere Sachverhalte als die, welche einer fahrlässigen Mittäterschaft zugrunde liegen.

5. Eine fahrlässige Mittäterschaft bzw. allgemeiner ausgedrückt eine Gesamthandlung bei einem Fahrlässigkeitsdelikt bedarf - wenn man das Konzept bejahten möchte - eines subjektiv verbindenden Beschlusses zur Ausführung der objektiv zum Erfolg führenden Handlung.

61 Trotz aller Weichenstellungen liess es das Bundesgericht offen, ob es eine fahrlässige Mittäterschaft gibt. Nur schon die Tatsache aber, dass das Bundesgericht den Fall nicht mit der Feststellung löst, es gäbe keine fahrlässige Mittäterschaft, sondern dass deren erstes Kriterium der gemeinsame Beschluss einer (sorgfaltswidrigen) Handlung - nicht nachgewiesen sei, darf die Befürworter der Rechtsfigur einer fahrlässigen Mittäterschaft optimistisch in die Zukunft blicken lassen. 